\title{
SOME CYCLIC INEQUALITIES
}

\author{
by V. J. BASTON
}

(Received 25th January 1973)

In this note we prove some cyclic inequalities which are generalisations of known results. We shall assume throughout that $a_{i+n}=a_{i} \geqq 0$ for all $i$, that no denominator in the statement of a result vanishes and finally that $p, m$ and $q$ are positive integers. We shall also use $A(i, m)$ to denote $\sum_{j=1}^{m} a_{i+j}$ with the convention that $A(i, 0)=0$. The most interesting of our results is probably Theorem 2 since, in the special case $p=1, m=2, r=0$, it gives a lower bound of $\frac{1}{3} n$ for the Shapiro sum $\sum_{i=1}^{n} \frac{a_{i}}{A(i, 2)}$. Although it is by no means best possible, see (2), our method implicitly gives a really simple way of obtaining this lower bound which, incidentally, is an improvement on Rankin's original result (5).

In (1) Boarder and Daykin established the following results:

$$
\begin{aligned}
& \inf _{n} \inf \frac{1}{n} \sum_{i=1}^{n} \frac{a_{i+1}+a_{i+4}}{A(i, 4)} \leqq \frac{1}{3}, \\
& \inf _{n} \inf \frac{1}{n} \sum_{i=1}^{n} \frac{a_{i+1}+a_{i+2}+a_{i+4}}{A(i, 4)} \leqq \frac{1}{2} \text {, } \\
& \inf _{n} \inf \frac{1}{n} \sum_{i=1}^{n} \frac{a_{i+1}+a_{i+2}+a_{i+4}}{a_{i+1}+a_{i+3}+a_{i+4}} \leqq \frac{1}{2} \text {, }
\end{aligned}
$$

where the second infs are evaluated over all choices of $a_{1}, \ldots, a_{n}$. It follows from Theorem 1 below that equality holds in all three cases; in the inequality for the upper bound, take $p=q=1, m=2$ for (1) and $p=2, m=1=q$ for (2) and (3).

\section{Theorem 1.}

$$
\frac{m}{\left[\frac{p+q+m-1}{n}\right]+1} \leqq \sum_{i=1}^{n} \frac{A(i+p, m)}{A(i, p+m+q)} \leqq \frac{m}{m+\min (p, q)} n
$$

where $\left[\frac{p+q+m-1}{n}\right]$ denotes the integral part of $\frac{p+q+m-1}{n}$.

Proof. For the lower bound let $w=\left[\frac{p+q+m-1}{n}\right]$ and $s=\sum_{i=1}^{n} a_{i}$ then 
$A(i, p+m+q) \leqq(w+1) s$ and we have

$$
\sum_{i=1}^{n} \frac{A(i+p, m)}{A(i, p+m+q)} \geqq \frac{1}{(w+1) s} \sum_{i=1}^{n} A(i+p, m)=\frac{m}{w+1} .
$$

Equality clearly holds when $p+m+q$ is a multiple of $n$. By considering, for large $x, \mathrm{a}_{i}=x^{i}$ when $1 \leqq i \leqq n$ the lower bound is also seen to be best possible when $p+m+q<n$.

We now prove the upper bound result. Let $k=\min (p, q)$. If for $a_{1}, \ldots, a_{n}$ there is an $i$ such that $A(i, m+k)=0$ then, by omitting an appropriate number of zeros, we can obtain a subsequence $b_{1}, \ldots, b_{r}$ such that

$$
\sum_{i=1}^{n} \frac{A(i+p, m)}{A(i, p+m+q)} \leqq \sum_{i=1}^{r} \frac{b_{i+p+1}+\ldots+b_{i+p+m}}{b_{i+p-k+1}+\ldots+b_{i+p+m+k}}
$$

where $b_{i+r}=b_{i}$ and $b_{i+1}+\ldots+b_{i+m+k}>0$ for all $i$. Hence we need only consider those $a_{1}, \ldots, a_{n}$ for which $A(i, m+k)>0$ for all $i$. Furthermore, since

$$
\sum_{i=1}^{n} \frac{A(i+p, m)}{A(i, p+m+q)} \leqq \sum_{i=1}^{n} \frac{A(i+k, m)}{A(i, m+2 k)}
$$

it is now sufficient to prove the result for the case when $p=q$.

Let $\quad p+m=e m+f$ where $0 \leqq f<m$,

$f_{r}$ be the integral part of $\frac{r f}{m}$,

$$
\begin{aligned}
& u_{0}=1 \text { and } u_{r}=\frac{r f}{m}-f_{r} \text { for } r \geqq 1, \\
& e_{0}=0 \text { and } e_{r}=e-1+u_{1}-u_{r}+u_{r-1} \text { for } r \geqq 1, \\
& v_{r}=\sum_{s=0}^{r} e_{s} .
\end{aligned}
$$

We then have $1-u_{r-1}+e_{r}+u_{r}=\frac{p+m}{m}$ for $r \geqq 1$,

$e_{r}$ is a non-negative integer for $r \geqq 0$,

$1 \leqq v_{r} \leqq p$ for $1 \leqq r<m$ and $v_{m}=p+1$.

Thus

$$
\begin{aligned}
& \frac{A(i+p, m)}{A(i, m+2 p)}=\frac{m}{p+m} \sum_{r=1}^{m} a_{i+p+r} \frac{1-u_{r-1}+e_{r}+u_{r}}{A(i, 2 p+m)} \\
& \leqq \frac{m}{+m} \sum_{r=1}^{m}\left\{\frac{\left(1-u_{r-1}\right) a_{i+p+r}}{A\left(i+p+1-v_{r-1}, p+m\right)}+\right. \\
&\left.\sum_{j=0}^{e_{r}} \frac{a_{i+p+r}}{A\left(i+p-j-v_{r-1}, p+m\right)}+\frac{u_{r} a_{i+p+r}}{A\left(i+p-v_{r}, p+m\right)}\right\} .
\end{aligned}
$$


Using

we have

$$
\sum_{i=1}^{n} \frac{a_{i+t}}{A(i-j, p+m)}=\sum_{i=1}^{n} \frac{a_{i+j+t}}{A(i, p+m)}
$$

$$
\begin{aligned}
& \sum_{i=1}^{n} \frac{A(i+p, m)}{A(i, 2 p+m)} \leqq \frac{m}{p+m} \sum_{r=1}^{m} \sum_{i=1}^{n} \frac{1}{A(i, p+m)}\left\{\left(1-u_{r-1}\right) a_{i+r+v_{r-1}-1}\right. \\
& \left.\quad+\sum_{j=0}^{e_{r}-1} a_{i+r+j+v_{r-1}}+u_{r} a_{i+r+v_{r}}\right\}=\frac{m}{p+m} \sum_{i=1}^{n} \frac{A(i, p+m)}{A(i, p+m)}=\frac{m}{p+m} n
\end{aligned}
$$

For the case $p=q$ we can see that the bound is attained when $n=r(p+m)$ by considering $a_{1+j(p+m)}=1$ for $j=0,1, \ldots, r-1$ and $a_{i}=0$ otherwise.

The next theorem is both a generalisation and a sharpening of an inequality of Diananda (3, Theorem 1).

Theorem 2.

$$
\frac{2 m}{2 m-p-r} n \leqq \sum_{i=1}^{n} \frac{A(i, p+m+r)}{A(i+p, m)} \text { if } p+r \leqq m \text {. }
$$

Proof. By repeated use of the arithmetic-geometric mean inequality we have, for $p+r \leqq m$,

$$
\begin{aligned}
\sum_{i=1}^{n} \frac{A(i, p+m+r)}{A(i+p, m)} & \geqq \sum_{i=1}^{n} \frac{A(i, p+m+r)}{A(i+p, m)} \frac{2\{A(i, m) \cdot A(i+p+r, m)\}^{\frac{1}{2}}}{A(i, m)+A(i+p+r, m)} \\
& \geqq 2 n\left\{\prod_{i=1}^{n} \frac{A(i, p+m+r)}{A(i, m)+A(i+p+r, m)}\right\}^{1 / n} \\
& =2 n\left\{\prod_{i=1}^{n}\left(1+\frac{A(i+p+r, m-p-r)}{A(i, p+m+r)}\right)\right\}^{-1 / n} \\
& \geqq 2 n^{2}\left\{n+\sum_{i=1}^{n} \frac{A(i+p+r, m-p-r)}{A(i, p+m+r)}\right\}^{-1} \\
& \geqq \frac{2 n^{2}}{n+\frac{m-p-r}{m} n}=\frac{2 m}{2 m-p-r} n \text { (by Theorem 1). }
\end{aligned}
$$

In generalising an inequality of Zulauf (6), Daykin (4) proved the following theorem for the special cases $m=1$ and $q=1$.

Theorem 3.

Proof.

$$
m \leqq \sum_{i=1}^{n} \frac{A(i, m)}{A(i, m+q)} \leqq n-q \text { if } m+q \leqq n
$$

$$
\begin{gathered}
\sum_{i=1}^{n} \frac{A(i, m)}{A(i, m+q)} \geqq \sum_{i=1}^{n} \frac{A(i, m)}{A(i, n)}=m \frac{A(i, n)}{A(i, n)}=m . \\
\sum_{i=1}^{n} \frac{A(i, m)}{A(i, m+q)}=\sum_{i=1}^{n}\left(1-\frac{A(i+m, q)}{A(i, m+q)}\right) \leqq n-q .
\end{gathered}
$$


For the upper bound consider, for large $x, a_{i}=0$ when $1 \leqq i \leqq m-1$ and $a_{i}=x^{n-i}$ when $m \leqq i \leqq n$. For the lower bound consider, for large $x, a_{i}=x^{i}$ when $1 \leqq i \leqq n$.

I am indebted to the referee for his comments which have been most helpful.

\section{REFERENCES}

(1) J. C. Boarder and D. E. Daykin, Inequalities for certain cyclic sums II, Proc. Edinburgh Math. Soc. 18 (1973), 209-218.

(2) P. H. Diananda, A cyclic inequality and an extension of it II, Proc. Edinburgh Math. Soc. 13 (1962), 143-152.

(3) P. H. Diananda, Some cyclic and other inequalities, Proc. Cambridge Philos. Soc. 58 (1962), 425-427.

(4) D. E. DaYkIN, Inequalities for functions of a cyclic nature, J. London Math. Soc. 3 (1971), 453-462.

(5) R. A. Rankin, A cyclic inequality, Proc. Edinburgh Math. Soc. 12 (1961), 139-147.

(6) A. Zulauf, Note on some inequalities, Math. Gaz. 43 (1959), 42-44.

THE UNIVERSITY

SOUTHAMPTON SO9 $5 \mathrm{NH}$ 